\title{
On Stability of Bases Made from Perturbed Exponential Systems in Morrey Type Spaces
}

\author{
Fatima Guliyeva \\ Institute of mathematics and Mechanics of NAS of Azerbaijan
}

Received: June 3, 2020. Revised: August 24, 2020. Accepted: August 28, 2020. Published: August 31, 2020.

\begin{abstract}
Perturbed exponential system $\left\{e^{i \lambda_{k} x}\right\}_{k \in Z}$ (where $\left\{\lambda_{n}\right\}$ is some sequence of real numbers) is considered in Morrey spaces $L^{p, \alpha}(0, \pi)$. These spaces are non-separable (except for exceptional cases), and therefore the above system is not complete in them. Based on the shift operator, we define the subspace $M^{p, \alpha}(0, \pi) \subset L^{p, \alpha}(0, \pi)$, where continuous functions are dense. We find a condition on the sequence $\left\{\lambda_{n}\right\}$, which is sufficient for the above system to form a basis for the subspace $M^{p, \alpha}(0, \pi)$. Our results are the analogues of those obtained earlier for the Lebesgue spaces $L_{p}$. We also establish an analogue of classical Levinson theorem on the completeness of above system in the spaces $L_{p}, 1 \leq p \leq+\infty$.
\end{abstract}

Keywords-system of exponent, basicity, perturbation, Morrey space

\section{INTRODUCTION}

Consider perturbed systems of sines

$$
\left\{\sin \lambda_{n} x\right\}_{n \in N}
$$

and cosines

$$
\left\{\cos \lambda_{n} x\right\}_{n \in Z_{+}}
$$

where $N$ is a set of all positive integers, $Z_{+}=\{0\} \cup N$, and $\left\{\lambda_{n}\right\} \subset R$ is some sequence of real numbers. These systems are the natural perturbations of classical systems of sines and cosines, and they are also the eigenfunctions of second order ordinary differential operator with integral boundary condition. Moreover, it should be noted that the frame theory originates from the research by Duffin R.J. and Schaeffer A.C. [1] dedicated to the frame properties of such systems in the spaces $L_{2}$. That's why there is great interest in studying basis properties of these systems in different kinds of function spaces. First results in this field belong probably to PaleyWiener [2] and N. Levinson [3]. The well-known Kadets 1/4 theorem also belongs to this field (see [4]). When $\lambda_{n}$ has a constant shift $\lambda_{n}=n+\alpha(\alpha \in R)$, the systems (1) and (2) arise in the solution of mixed or elliptic type differential equations by the Fourier method (see, e.g., [5-7]. In view of this, many authors have studied the basis properties of the systems (1) and (2) (see, e.g., [5;8-16]). All above-mentioned works treat basis properties in the Lebesgue spaces.

Recently there has been increasing interest in non-standard function spaces in the context of applications to the problems of mechanics and mathematical physics. Among those spaces, we can mention Lebesgue spaces with variable summability index, Morrey spaces, grand-Lebesgue spaces, etc. Some relevant studies can be found in [17-20]. Each of these spaces has its own differences compared to classical Lebesgue type spaces. For example, Morrey and grand-Lebesgue spaces are not separable, and therefore, a countable system of elements of course cannot be complete in these spaces. So one has to choose reasonable subspaces of these spaces to treat some approximation problems. In the context of classical trigonometric systems and their linear perturbations (with regard to a phase), approximation problems in these spaces have been studied in varying degrees (for more details see, e.g., $[16 ; 21-26])$.

In this work, we consider a perturbed exponential system $\left\{e^{i \lambda_{k} x}\right\}_{k \in Z}$ in the Morrey space $L^{p, \alpha}(0, \pi), 1<p<+\infty$, $0<\alpha<1$. Based on the continuity of the shift operator, we define a subspace $M^{p, \alpha}(0, \pi) \subset L^{p, \alpha}(0, \pi)$ of this space. We establish an analogue of classical Levinson theorem on the replacement of a finite number of elements of this system by other elements. We find a condition on the sequence $\left\{\lambda_{n}\right\}$, which is sufficient for this system to form a basis for $M^{p, \alpha}(0, \pi)$. Our results are the analogues of the corresponding results obtained for Lebesgue spaces (see, e.g., [27]).

\section{NEEDFUL INFORMATION}

In this section, we state some needful concepts and facts to be used to obtain our main results. Let's first define the Morrey space on $(a, b)$. It is a Banach space of all measurable functions over $(a, b)$ with the finite norm 


$$
\|f\|_{L^{p, \alpha}(a, b)}=\sup _{I \subset(a, b)}\left(|I|^{\alpha-1} \int_{I}|f(t)|^{p} d t\right)^{\frac{1}{p}},
$$

where sup is taken over all intervals $I \subset(a, b)$ and $|I|$ is a length of the interval $I$. It is easy to see that $L^{p, 1}(a, b)=L_{p}(a, b)$ and $L^{p, 0}(a, b)=L_{\infty}(a, b)$. Moreover, the continuous embedding $L^{p, \alpha}(a, b) \subset L_{p}(a, b)$, $1 \leq p<+\infty, 0<\alpha \leq 1$, and the inequality

$$
\|f\|_{L_{p}(a, b)} \leq(b-a) \frac{1}{p(\alpha-1)}\|f\|_{L^{p, \alpha}(a, b)}, \quad \forall f \in L^{p, \alpha}(a, b)
$$

hold. Let

$$
\begin{gathered}
M^{p, \alpha}(a, b)=\left\{f \in L^{p, \alpha}(a, b):\right. \\
\left.\|f(\cdot+\delta)-f(\cdot)\|_{L^{p, \alpha}(a, b)} \rightarrow 0, \delta \rightarrow 0\right\} .
\end{gathered}
$$

$M^{p, \alpha}(a, b)$ for $1 \leq p<+\infty, 0<\alpha \leq 1$, is a separable Banach space and $C_{0}^{\infty}(a, b)$ (a space of infinitely differentiable and finite supported functions over $(a, b))$ is dense in it. When defining the space $M^{p, \alpha}(a, b)$, the function is assumed to be extended outside the interval $(a, b)$ by zero.

Definition 1. A system $\left\{f_{n}\right\}_{n \in N} \subset L^{p, \alpha}(a, b)$ is called $q$ Hilbert $(q>0)$ if there exists $c>0$ such that for every finite set of complex numbers $\left\{c_{n}\right\}$ the inequality

$$
\left\|\left\{c_{n}\right\}\right\|_{l_{q}} \leq c\left\|\sum_{n} c_{n} f_{n}\right\|_{L^{p, \alpha}(a, b)},
$$

holds.

Definition 2. A sequence $\left\{\lambda_{n}\right\}$ is called divided if $\inf _{i \neq j}\left|\lambda_{i}-\lambda_{j}\right|>0$.

We will also need the following

Statement 1. Suppose a finite number of elements in the basis of some Banach space are replaced by the other elements of this space. Then the following properties are equivalent for the newly obtained system:

i) it forms a basis;

ii) it is complete;

iii) it is minimal.

We will also use some concepts and facts from the theory of Banach function spaces. Let's state needful facts from this theory.

Let $(M ; / / ; \mu)$ be a measurable space with a measure. Denote a set of all measurable functions $f: M \rightarrow C$ (where $C$ is a complex plane) by $g$. Denote a subspace of functions from $\mathscr{y}$, which take on values $\mu$-a.e., by $\mathscr{Y}_{0}$. Let $\mathscr{F}^{+}=\{f \in \mathscr{F}: f \geq 0\}$.

Definition 3. A mapping $\rho: \mathscr{F}^{+} \rightarrow[0,+\infty]$ is called a Banach function norm (or simply a b.f.n.) if, for all $f, g, f_{n} \in \mathscr{F}^{+}, \forall n \in N$, for all constants $a \geq 0$ and for all $\mu$-measurable subsets $E \in \mathscr{M}$, the following properties hold:

$$
\begin{aligned}
& \text { (p1) } \rho(f)=0 \Leftrightarrow f=0 \quad \mu \text {-a.e; } \rho(\text { af })=a \rho(f) ; \\
& \quad \rho(f+g) \leq \rho(f)+\rho(g) ; \\
& \text { (p2) } 0 \leq g \leq f \quad \mu \text {-a.e. } \Rightarrow \rho(g) \leq \rho(f) ; \\
& \text { (p3) } 0 \leq f_{n} \uparrow f \quad \mu \text {-a.e. } \Rightarrow \rho\left(f_{n}\right) \uparrow \rho(f) ; \\
& \text { (p4) } \mu(E)<+\infty \Rightarrow \rho\left(\chi_{E}\right)<+\infty ; \\
& \text { (p5) } \mu(E)<+\infty \quad \Rightarrow \exists c_{E}>0: \quad \int_{E} f d \mu \leq c_{E} \rho(f),
\end{aligned}
$$

$\forall f \in \mathscr{F}^{+}$.

Definition 4. Let $\rho$ be a b.f.n.. The collection $X=X(\rho)$ of all functions $f \in \mathscr{G}$, for which $\rho(|f|)<+\infty$, is called a Banach function space (or simply a b.f.s.). For $f \in X$ define $\|f\|_{X}=\rho(|f|)$.

Definition 5. Suppose $f \in \mathscr{F}_{0}$. The decreasing rearrangement of $f$ is the function $f^{*}$ defined on $[0,+\infty)$ by

$$
f^{*}(t)=\inf \left\{\lambda: \mu_{f}(\lambda) \leq t\right\}, t \geq 0,
$$

where $\mu_{f}(\lambda)=\mu\{t:|f(t)|>\lambda\}, \lambda \geq 0$, is a distribution function of $t$.

Definition 6. Let $X$ be a b.f.s. The closure of the set of simple functions $M_{s}$ in $X$ is denoted by $X_{b}$.

Let $X$ be a b.f.s. over $(M ; \mu)$. Let

$$
\rho^{\prime}(g)=\sup \left\{\int_{M} f g d \mu: f \in \mathscr{F}^{+} ; \rho(f) \leq 1\right\}, \forall g \in \mathscr{F}^{+} \text {. }
$$

A space

$$
X^{\prime}=\left\{g \in \mathscr{F}: \rho^{\prime}(|g|)<+\infty\right\},
$$

is called an associate space (Kothe dual) of $X$.

The functions $f ; g \in \mathscr{F}_{0}$ are called equimeasurable if $\mu_{f}(\lambda)=\mu_{g}(\lambda), \quad \forall \lambda \geq 0$. Banach function $\rho: \mathscr{F}^{+} \rightarrow$ $[0,+\infty]$ is called rearrangement invariant if for arbitrary equimeasurable functions $f ; g \in \mathscr{F}^{+}$the relation $\rho(f)=\rho(g)$ holds. In this case, Banach function space $X$ with the norm $\|\cdot\|_{X}=\rho(|\cdot|)$ is said to be rearrangement invariant function space (r.i.s. for short). Classical Lebesgue, Orlicz, Lorentz, Lorentz-Orlicz spaces are r.i.s..

Definition 7. Let $X$ be a r.i.s. over a resonant space $(M ; \mu)$. For each finite value of $t$ belonging to the range of $\mu$, let $E \in \mathscr{M}: \mu(E)=t$ and

$$
\varphi_{X}(t)=\left\|\chi_{E}\right\|_{X} .
$$

The function $\varphi_{X}$ is called the fundamental function of $X$.

For resonant space see, e.g., [23, p.45]. 
Definition 8. A function $f$ in a b.f.s. $X$ is said to have absolutely continuous norm in $X$ if $\left\|f \chi_{E_{n}}\right\|_{X} \rightarrow 0$ for every sequence $\left\{E_{n}\right\} \subset \mathbb{l}: E_{n} \rightarrow \varnothing \quad \mu$-a.e.. The set of all functions in $X$ of absolutely continuous norm is denoted by $X_{a}$.

We also need the following theorems from the monograph [28].

Theorem 1 [28]. The subspaces $X_{a}$ and $X_{b}$ coincide if and only if the characteristic function $\chi_{E}$ has absolutely continuous norm for every set $E \in \mathscr{M}$ of finite measure.

Theorem 2. The Banach space dual $X^{*}$ of a b.f.s. $X$ is canonically isometrically isomorphic to the associate space $X^{\prime}$ if and only if $X$ has absolutely continuous norm.

We will also use the following statement from [28, p.14].

Statement 2. Let $X$ be a b.f.s. over $(M ; \mu)$ with norm $\|\cdot\|_{X}$. A function $f \in X$ has absolutely continuous norm if and only if $\left\|f \chi_{E_{n}}\right\| \downarrow 0$ for every sequence $\left\{E_{n}\right\}_{n \in N}$ satisfying $E_{n} \downarrow \varnothing \quad \mu$-a.e.

For more details about these facts see, e.g., [28].

\section{SUFFICIENT CONDITION FOR DIVIDEDNESS OF $\left\{\lambda_{n}\right\}$}

The following simple lemma is true.

Lemma 1. Let $\left\{\lambda_{n}\right\}_{n \in Z} \subset R$ be some sequence of real numbers. If the system $\left\{e^{i \lambda_{n} t}\right\}_{n \in Z}$ is $q$-Hilbert in $L^{p, \alpha}(-\pi, \pi), \quad 1 \leq p<+\infty, 0<\alpha \leq 1$, then $\left\{\lambda_{n}\right\}_{n \in Z}$ is divided.

Proof. From the definition of $q$-Hilbertness, we obtain

$$
2^{\frac{1}{q}} \leq c\left\|e^{i \lambda_{n} t}-e^{i \lambda_{k} t}\right\|_{L^{p, \alpha}(-\pi, \pi)}
$$

Taking into account the inequality

$$
\left|e^{i \lambda_{n} t}-e^{i \lambda_{k} t}\right|=2\left|\sin \frac{\lambda_{n}-\lambda_{k}}{2} t\right| \leq\left|\lambda_{n}-\lambda_{k}\right||t| \leq \pi\left|\lambda_{n}-\lambda_{k}\right|,
$$

we have

$$
\begin{aligned}
& \left|e^{i \lambda_{n} t}-e^{i \lambda_{k} t}\right|_{L^{p, \alpha}(-\pi, \pi)} \leq \\
& \pi\left|\lambda_{n}-\lambda_{k}\right|\left|1 \|_{L^{p, \alpha}(-\pi, \pi)}=\pi(2 \pi)^{\frac{\alpha}{p}}\right| \lambda_{n}-\lambda_{k} \mid .
\end{aligned}
$$

The rest follows directly from (3).

The lemma is proved.

The lemma below can be proved in exactly the same way.

Lemma 2. Let $\left\{\lambda_{n}\right\}_{n \in N} \subset R$ be some sequence. If the system (1) (or (2)) is $q$-Hilbert in $L^{p, \alpha}(0, \pi), 1 \leq p<+\infty$, $0<\alpha \leq 1$, then $\left\{\lambda_{n}\right\}_{n \in N}$ is divided.

\section{THE $M^{p, \alpha}$-ANALOGUE OF LEVINSON THEOREM}

In this section, we establish an analogue of Levinson theorem in $M^{p, \alpha}$. Denote by $\left(M^{p, \alpha}(a, b)\right)^{\prime}$ the associate space of $\left(M^{p, \alpha}(a, b)\right)$, i.e.

$$
\left(M^{p, \alpha}(a, b)\right)^{\prime}=\left\{g \in \mathscr{F}(a, b): \rho_{p, \alpha}^{\prime}(|g|)<+\infty\right\},
$$

where

$$
\rho_{p, \alpha}^{\prime}(g)=\sup \left\{\int_{a}^{b} f g d t: f \in \mathscr{F}^{+}(a, b) ;\|f\|_{L^{p, \alpha}(a, b)} \leq 1\right\},
$$

$F(a, b)$ are Lebesgue-measurable functions on $(a, b)$ and $\mathscr{F}^{+}(a, b)=\{f \in \mathscr{F}(a, b): f \geq 0\}$.

The following analogue of Levinson theorem is true.

Theorem 3. Let $\left\{\lambda_{k}\right\}_{k \in N} \subset C$ be some sequence. In order for the exponential system $\left\{e^{i \lambda_{k} t}\right\}_{k \in N}$ to be not complete in $M^{p, \alpha}(-\pi, \pi), 1 \leq p<+\infty, 0<\alpha \leq 1$, it is necessary and sufficient that there exist an entire function $F(\lambda)$ vanishing at all points $\lambda_{k}, k \in N$, and admitting representation

$$
F(\lambda)=\int_{-\pi}^{\pi} e^{i \lambda t} \overline{\psi(t)} d t
$$

where $\psi \in\left(M^{p, \alpha}(-\pi, \pi)\right)^{\prime}$ is some function.

Proof. Let the system $\left\{e^{i \lambda_{k} t}\right\}_{k \in N}$ be not complete in $M^{p, \alpha}(-\pi, \pi), 1 \leq p<+\infty, 0<\alpha \leq 1$. Then it is clear that there exists a non-zero functional $\vartheta \in\left(M^{p, \alpha}(-\pi, \pi)\right)^{*}$ such that

$$
\vartheta\left(e^{i \lambda_{k} t}\right)=0, \quad \forall k \in N .
$$

Let's show that the spaces $\left(M^{p, \alpha}(-\pi, \pi)\right)^{\prime}$ and $\left(M^{p, \alpha}(-\pi, \pi)\right)^{*}$ are isometrically isomorphic, i.e. they can be equated with each other. By Theorem 2, to show this, it suffices to prove that $M^{p, \alpha}(-\pi, \pi)$ has absolutely continuous norm. Let $f \in M^{p, \alpha}(-\pi, \pi)$ be an arbitrary function. As $C[-\pi, \pi]$ (a space of continuous functions on $[-\pi, \pi])$ is dense in $M^{p, \alpha}(-\pi, \pi)$, for $\forall \varepsilon>0$, $\exists f_{0} \in C[-\pi, \pi]$ we have

$$
\left\|f-f_{0}\right\|_{L^{p, \alpha}(-\pi, \pi)}<\varepsilon .
$$

Let $\left\{E_{n}\right\}_{n \in N} \subset(-\pi, \pi)$ be an arbitrary sequence of (Lebesgue) measurable sets such that $E_{n} \downarrow \varnothing m$-a.e. ( $m$ is a Lebesgue measure). Recall that $E_{n} \downarrow \varnothing m$-a.e. means that $\chi_{E_{n}} \downarrow 0 \quad m$-a.e.. Let's show that $\left\|f \chi_{E_{n}}\right\|_{L^{p, \alpha}(-\pi, \pi)} \downarrow 0$. So, let $\varepsilon>0$ be an arbitrary number. We have 


$$
\begin{aligned}
& \left\|f \chi_{E_{n}}\right\|_{L^{p, \alpha}(-\pi, \pi)} \leq\left\|\left(f-f_{0}\right) \chi_{E_{n}}\right\|_{L^{p, \alpha}(-\pi, \pi)}+ \\
& \left\|f_{0} \chi_{E_{n}}\right\|_{L^{p, \alpha}(-\pi, \pi)}<\varepsilon+\left\|f_{0} \chi_{E_{n}}\right\|_{L^{p, \alpha}(-\pi, \pi)} .
\end{aligned}
$$

Let $c=\left\|f_{0}\right\|_{L_{\infty}(-\pi, \pi)}$. We have

$$
\left\|f_{0} \chi_{E_{n}}\right\|_{L^{p, \alpha}(-\pi, \pi)} \leq c\left\|\chi_{E_{n}}\right\|_{L^{p, \alpha}(-\pi, \pi)}=c\left|E_{n}\right|^{\frac{\alpha}{p}},
$$

where $|\cdot|$ is a Lebesgue measure. Obviously, $\lim _{n} E_{n}=$ $\bigcap_{n} E_{n}=\varnothing m$-a.e.. Consequently,

$$
\lim _{n}\left|E_{n}\right|=\left|\lim _{n} E_{n}\right|=0 .
$$

Then from (4) it follows that $\left\|f \chi_{E_{n}}\right\|_{L^{p, \alpha}(-\pi, \pi)} \rightarrow 0, n \rightarrow \infty$.

Thus, by Statement 2 , the space $M^{p, \alpha}(-\pi, \pi)$ has absolutely continuous norm. Then from Theorem 2 it follows that $\left(M^{p, \alpha}(-\pi, \pi)\right)^{*}=\left(M^{p, \alpha}(-\pi, \pi)\right)^{\prime}$. Hence, it is clear that $\quad \exists \psi \in\left(M^{p, \alpha}(-\pi, \pi)\right)^{\prime}: \vartheta(f)=\int_{-\pi}^{\pi} f(t) \overline{\psi(t)} d t$, $\forall f \in\left(M^{p, \alpha}(-\pi, \pi)\right)$. Let

$$
F(\lambda)=\int_{-\pi}^{\pi} e^{i \lambda t} \overline{\psi(t)} d t, \lambda \in C .
$$

Obviously, $F($.$) is an entire function and F\left(\lambda_{k}\right)=0$, $\forall k \in N$.

The theorem is proved.

Theorem 4. If the entire function $F($.$) is represented in the$ form (5), $\psi \in\left(M^{p, \alpha}(-\pi, \pi)\right)^{\prime}, \quad 1 \leq p<+\infty, \quad 0<\alpha \leq 1$, $F\left(\lambda_{0}\right)=0$, and $\mu \in C$ is an arbitrary number, then the function

$$
F_{1}(\lambda)=\frac{\lambda-\mu}{\lambda-\lambda_{0}} F(\lambda)
$$

is also represented in the form (5). let

Proof. Absolutely similar to the proof of Levinson theorem,

$$
\overline{\varphi(x)}=\overline{\psi(x)}+i\left(\mu-\lambda_{0}\right) e^{-i \lambda_{0} x} \int_{-\pi}^{x} e^{i \lambda_{0} y} \overline{\psi(y)} d y .
$$

By multiplying both sides by $e^{i \lambda x}$ and integrating from $-\pi$ to $\pi$, we obtain

$$
\int_{-\pi}^{\pi} e^{i \lambda x} \overline{\varphi(x)} d x=F(\lambda)+i\left(\mu-\lambda_{0}\right) \int_{-\pi}^{\pi} e^{i\left(\lambda-\lambda_{0}\right) x}\left(\int_{-\pi}^{x} e^{i \lambda_{0} y} \overline{\psi(y)} d y\right) d x
$$

Changing the order of integration, we have

$$
\begin{aligned}
& \int_{-\pi}^{\pi} e^{i \lambda x} \overline{\varphi(x)} d x=F(\lambda)+i\left(\mu-\lambda_{0}\right) \int_{-\pi y}^{\pi} \int_{0}^{\pi} e^{i\left(\lambda-\lambda_{0}\right) x} e^{i \lambda_{0} y} \overline{\psi(y)} d x d y= \\
& =F(\lambda)+\frac{\mu-\lambda_{0}}{\lambda-\lambda_{0}} \int_{-\pi}^{\pi}\left(e^{i\left(\lambda-\lambda_{0}\right) \pi}-e^{i\left(\lambda-\lambda_{0}\right) y}\right) e^{i \lambda_{0} y} \overline{\psi(y)} d y= \\
& =F(\lambda)-\frac{\mu-\lambda_{0}}{\lambda-\lambda_{0}} \int_{-\pi}^{\pi} e^{i \lambda y} \overline{\psi(y)} d y=\frac{\lambda-\mu}{\lambda-\lambda_{0}} F(\lambda)=F_{1}(\lambda),
\end{aligned}
$$

i.e.

$$
F_{1}(\lambda)=\int_{-\pi}^{\pi} e^{i \lambda x} \overline{\varphi(x)} d x .
$$

In order to the represent the function $\varphi(x)$ by a functional (bounded) on $M^{p, \alpha}(-\pi, \pi)$, it is sufficient to show that $\varphi \in\left(M^{p, \alpha}(-\pi, \pi)\right)^{\prime} . \quad$ It is absolutely clear that $\left|e^{i \lambda_{0} x}\right| \leq$ const $<+\infty, \forall x \in[-\pi, \pi]$. Therefore, from the expression (6) for $\varphi(\cdot)$, it follows that it now suffices to prove that $\int_{-\pi}^{x}|\psi(y)| d y \in\left(M^{p, \alpha}(-\pi, \pi)\right)^{\prime}$. But this is obvious, because $\int_{-\pi}^{x}|\psi(y)| d y \in C[-\pi, \pi]$.

The theorem is proved.

This theorem has the following direct corollary.

Corollary 1. Let the system $\left\{e^{i \lambda_{k} x}\right\}_{k \in N}$ be complete in $M^{p, \alpha}(-\pi, \pi), \quad 1 \leq p<+\infty, \quad 0<\alpha \leq 1$. If $n$ arbitrary functions are removed from this system and $n$ other functions $e^{i \mu_{j} x}, j=\overline{1, n}$, where $\mu_{1}, \ldots, \mu_{n}$ are arbitrary complex numbers different from any of $\lambda_{k}$, are added instead of them, then the newly obtained system will be complete in $M^{p, \alpha}(-\pi, \pi)$.

\section{ON STABILITY OF EXPONENTIAL BASES IN $M^{p, \alpha}$}

The following main theorem is true.

Theorem 5. Let $\left\{\lambda_{n}\right\}_{n \in Z} ; \quad\left\{\mu_{n}\right\}_{n \in Z} \subset R$ be some sequences, $\lambda_{i} \neq \lambda_{j}, \mu_{i} \neq \mu_{j}$ for $i \neq j$. Let

$$
\sum_{n=-\infty}^{+\infty}\left|\lambda_{n}-\mu_{n}\right|^{\beta}<+\infty
$$

where $\beta=\min (p, q), \frac{1}{p}+\frac{1}{q}=1$ and $p \in(1,+\infty)$ is some number. If the system $\left\{e^{i \lambda_{n} x}\right\}_{n \in Z}$ forms a basis for $M^{p, \alpha}(-\pi, \pi), 0<\alpha \leq 1$, equivalent to the basis $\left\{e^{i n x}\right\}_{n \in Z}$, 
then the system $\left\{e^{i \mu_{n} x}\right\}_{n \in Z}$ also forms a basis for $M^{p, \alpha}(-\pi, \pi)$, equivalent to $\left\{e^{i n x}\right\}_{n \in Z}$.

Proof. We first consider the case $1<p \leq 2$. Then it is clear that $q \geq 2$ and $\beta=p$. Let $\varphi_{n}(x)=e^{i \lambda_{n} x}$, $\psi_{n}(x)=e^{i \mu_{n} x}$. We have

$$
\left\|\varphi_{n}-\psi_{n}\right\|_{L^{p, \alpha}(-\pi, \pi)}^{p} \leq c\left|\lambda_{n}-\mu_{n}\right|^{p},
$$

where $c>0$ is a constant independent of $n$. Consequently,

$$
\sum_{n=-\infty}^{+\infty}\left\|\varphi_{n}-\psi_{n}\right\|_{L^{p, \alpha}(-\pi, \pi)}^{p}<+\infty
$$

Let $\left\{c_{n}\right\}$ be an arbitrary finite set of numbers $c_{n} \in C$. Then from the Hausdorff-Young theorem we obtain

$$
\left\|\left\{c_{n}\right\}\right\|_{l_{q}} \leq c\left\|\sum_{n} c_{n} e^{i n x}\right\|_{L_{p}(-\pi, \pi)},
$$

where $c>0$ is a constant independent of $c_{n}$. In what follows, by $c$ we will denote absolute constants (which can be different in different places). Then we have

$$
\left\|\left\{c_{n}\right\}\right\|_{l_{q}} \leq c\left\|\sum_{n} c_{n} e^{i n x}\right\|_{L^{p, \alpha}(-\pi, \pi)} .
$$

As the bases $\left\{\varphi_{n}\right\}_{n \in Z}$ and $\left\{e^{i n x}\right\}_{n \in Z}$ are equivalent, from (7) it follows

$$
\left\|\left\{c_{n}\right\}\right\|_{l_{q}} \leq c\left\|\sum_{n} c_{n} \varphi_{n}(x)\right\|_{L^{p, \alpha}(-\pi, \pi)} .
$$

Consider some number $m \in N$ and let

We have

$$
f_{n}= \begin{cases}\varphi_{n}, & |n|<m \\ \psi_{n}, & |n| \geq m\end{cases}
$$

$$
\begin{gathered}
\left\|\sum_{n} c_{n}\left(f_{n}-\varphi_{n}\right)\right\|_{L^{p, \alpha}(-\pi, \pi)} \leq \sum_{n} \mid c_{n}\left\|f_{n}-\varphi_{n}\right\|_{L^{p, \alpha}(-\pi, \pi)} \leq \\
\leq\left\|\left\{c_{n}\right\}\right\|_{l_{q}}\left(\sum_{n}\left\|f_{n}-\varphi_{n}\right\|_{L^{p, \alpha}(-\pi, \pi)}^{p}\right)^{\frac{1}{p}} \leq \\
\leq c\left(\sum_{|n| \geq m}\left\|\varphi_{n}-\psi_{n}\right\|_{L^{p, \alpha}(-\pi, \pi)}^{p}\right)^{\frac{1}{p}}\left\|\sum_{n} c_{n} \varphi_{n}\right\|_{L^{p, \alpha}(-\pi, \pi)}= \\
=c(m)\left\|\sum_{n} c_{n} \varphi_{n}\right\|_{L^{p, \alpha}(-\pi, \pi)},
\end{gathered}
$$

where

$$
c(m)=c\left(\sum_{|n| \geq m}\left\|\varphi_{n}-\psi_{n}\right\|_{L^{p, \alpha}(-\pi, \pi)}^{p}\right)^{\frac{1}{p}} .
$$

It is absolutely clear that $\lim _{m \rightarrow \infty} c(m)=0$, and therefore, for large $m$ we have $0<c(m)<1$. Then from the Paley-Wiener theorem (for Banach case; see, e.g., [29, p.187]) and the relation (8) it follows that the system $\left\{f_{n}\right\}_{n \in Z}$ forms a basis for $M^{p, \alpha}(-\pi, \pi)$, equivalent to $\left\{\varphi_{n}\right\}_{n \in Z}$. From the completeness of the system $\left\{f_{n}\right\}_{n \in Z}$ in $M^{p, \alpha}(-\pi, \pi)$ and Corollary 1 it follows that the system $\left\{\psi_{n}\right\}_{n \in Z}$ is also complete in $M^{p, \alpha}(-\pi, \pi)$. Then, by Statement 1, the system $\left\{\psi_{n}\right\}_{n \in Z}$ also forms a basis for $M^{p, \alpha}(-\pi, \pi)$, equivalent to $\left\{\varphi_{n}\right\}_{n \in Z}$.

Now let's consider the case $p>2$. Then we have $q<2$ and $\quad \beta=q$. In this case the embedding $M^{p, \alpha}(-\pi, \pi) \subset M^{q, \alpha}(-\pi, \pi)$ holds, i.e. $\|f\|_{L^{q, \alpha}(-\pi, \pi)} \leq$ $c\|f\|_{L^{p, \alpha}(-\pi, \pi)}, \quad \forall f \in M^{p, \alpha}(-\pi, \pi)$.

For a finite set $\left\{c_{n}\right\}$ we have

$$
\left\|\sum_{n} c_{n}\left(f_{n}-\varphi_{n}\right)\right\|_{L^{p, \alpha}(-\pi, \pi)} \leq\left\|\left\{c_{n}\right\}\right\|_{l_{p}}\left(\sum_{|n| \geq m}\left\|\psi_{n}-\varphi_{n}\right\|_{L^{p, \alpha}(-\pi, \pi)}^{q}\right)^{\frac{1}{q}} \leq
$$$$
\begin{aligned}
& \leq c\left(\sum_{|n| \geq m}\left\|\psi_{n}-\varphi_{n}\right\|_{L^{p, \alpha}(-\pi, \pi)}^{q}\right)^{\frac{1}{q}}\left\|\sum_{n} c_{n} e^{i n x}\right\|_{L_{q}(-\pi, \pi)} \leq \\
& \leq c\left(\sum_{|n| \geq m}\left\|\psi_{n}-\varphi_{n}\right\|_{L^{p, \alpha}(-\pi, \pi)}^{q}\right)^{\frac{1}{q}}\left\|\sum_{n} c_{n} e^{i n x}\right\|_{L_{p}(-\pi, \pi)} \leq
\end{aligned}
$$$$
\leq c\left(\sum_{|n| \geq m}\left\|\psi_{n}-\varphi_{n}\right\|_{L^{p, \alpha}(-\pi, \pi)}^{q}\right)^{\frac{1}{q}}\left\|\sum_{n} c_{n} e^{i n x}\right\|_{L^{p, \alpha}(-\pi, \pi)} \leq
$$$$
\leq c\left(\sum_{|n| \geq m}\left\|\psi_{n}-\varphi_{n}\right\|_{L^{p, \alpha}(-\pi, \pi)}^{q}\right)^{\frac{1}{q}}\left\|\sum_{n} c_{n} \varphi_{n}\right\|_{L^{p, \alpha}(-\pi, \pi)} .
$$

Proceeding absolutely similar to the case $p \leq 2$, we now establish the basicity of the system $\left\{\psi_{n}\right\}_{n \in Z}$ for $M^{p, \alpha}(-\pi, \pi)$.

The theorem is proved.

\section{CONCLUSION}

In conclusion it should be noted that the perturbed exponential system which arise in solving differential 
equations by Fourier method is considered. Method of establishing the basicity of these systems takes its origin from the classical work by Paley-Wiener. $L_{p}$-theory of this direction well developed and more details on concerning results can be found in [29]. In this work we consider non separable case, namely the Morrey space which is of particular interest from the point of view of the theory of differential equations. More details on related topics can be found in [30-32]. We have obtained results concerning Morrey space similar to $L_{p}$ - theory. Generally speaking, the fact on basicity of these systems does not hold true for Morrey space. We defined a suitable subspace of Morrey space and established sufficient conditions for the basicity of the considered perturbed exponential system in this subspace. The obtained results can be used in solving of differential equations, in approximation theory and in spectral theory of differential operators.

\section{REFERENCES}

[1] R.J. Duffin, A.C. Schaeffer, A class of nonharmonic Fourier series, Trans. Amer. Math. Soc., vol. 72, 1952, pp. $341-366$.

[2] R. Paley, N. Wiener, Fourier Transforms in the Complex Domain. Amer. Math. Soc. Colloq. Publ., 19 (Amer. Math. Soc., RI, 1934).

[3] B.Y. Levin, Distribution of Roots of Entire Functions. Moscow, GITL, 1956 (in Russian).

[4] M.I. Kadets, On the exact value of Paley-Wiener's constant. DAN SSSR, 1964 (in Russian).

[5] S.M. Ponomarev, On one eigenvalue problem. DAN SSSR, vol. 249, №5, 1979, pp. 1068-1070 (in Russian).

[6] E.I. Moiseev, On some boundary value problems for mixed type equations. Diff. uravneniya, v. 28, №1, 1992, 123-132 (in Russian)

[7] E.I. Moiseev, On solution of the Frankl problem in a special domain. Diff. uravn., vol. 28, №4, 1992, pp. 682692 (in Russian).

[8] E.I. Moiseev, On basicity of sine and cosine systems. DAN SSSR, vol. 275, № 4, 1984, pp. 794-798 (in Russian).

[9] E.I. Moiseev, On basicity of one sine system. Diff. uravneniya, vol. 23, № 1, 1987, pp. 177-179 (in Russian).

[10]A.M. Sedletski, On convergence of non-harmonic Fourier series in exponential, sine and cosine systems. DAN SSSR, vol. 301, №5, 1988, pp. 501-504 (in Russian).

[11]B.T. Bilalov, Basicity of some exponential, sine and cosine systems. Dif. uravneniya, vol. 26, №1, 1990, pp. 10-16 (in Russian).

[12] B.T. Bilalov, Bases from exponents, cosines and sines which are the eigenvalues of differential operators. Dif. Ur., vol. 39, №5, 2003, pp. 1-5 (in Russian).

[13]B.T. Bilalov, Basis properties of some exponential, cosine and sine systems. Sibirskiy matem. jurnal, vol. 45, №2, 2004, pp. 264-273 (in Russian).
[14] B.T. Bilalov, On a remark on Riesz basicity of systems of sines and cosines, Trans. Sci. IMM NAS Az., vol. XXII, №. 4, 2002, pp. 47-50.

[15]B.T. Bilalov, Bases in $L_{p}$ from exponents, cosines and sines, Trans. Sci. IMM NAS Az., No. 1, 2002, pp. 45-51.

[16]B.T. Bilalov, Z.G. Guseynov, Basicity of a system of exponents with a piece-wise linear phase in variable spaces, Mediterr. J. Math., vol. 9 , № 3, 2012, pp.487498.

[17] Shanli Ye, Products of Volterra-type operators and composition operators from analytic Morrey spaces into Zygmund spaces, WSEAS Transactions on Mathematics, pp. 378-388, Volume 18, 2019.

[18] Michael Gil, Exponential Stability of Nonautonomous Infinite Dimensional Systems, WSEAS Transactions on Mathematics, pp. 80-84, Volume 17, 2018.

[19]F.A. Guliyeva, S.R. Sadigova, On Some Properties of Convolution in Morrey Type Spaces. Azerb. J Math., vol. 8, №. 1, 2018, pp. 140-150

[20] L. Caso, R. D’Ambrosio, L. Softova,Generalized Morrey Spaces over Unbounded Domains. Azerb. J Math., vol. 10, №. 1, 2020, pp. 193-208

[21] B.T. Bilalov, F.A. Guliyeva, A completeness criterion for a double system of powers with degenerate coefficients. Sib. Mat.1 Journ., vol.54, №. 3, 2013, pp. 536-543.

[22] B.T. Bilalov, The basis property of a perturbed system of exponentials in Morrey-type spaces, Sib. Math. Journ., vol. 60, №.2, 2019, pp. 323-350.

[23]B.T. Bilalov, Z.G. Guseynov, Criterion for basicity of perturbed exponential system for Lebesgue spaces with variable index of summability. Dokladi Akademii Nauk, 2011, t.436, №5, pp.586-589 (in Russian)

[24]B.T. Bilalov, A.A. Huseynli, S.R. El-Shabrawy, Basis Properties of Trigonometric Systems in Weighted Morrey Spaces. Azerb. J Math., vol. 9, №. 2, 2019, pp. 183-209.

[25] B.T. Bilalov, F.Sh. Seyidova, Basicity of a system of exponents with a piecewise linear phase in Morrey-type spaces, Turk. J Math., vol. 43, 2019, pp.1850 - 1866.

[26] N.P. Nasibova, F.Sh. Seyidova, On the basicity of a perturbed system of exponents with a unit in Morrey-type spaces. Trans. Natl. Acad. Sci. Azerb. Ser. Phys.-Tech. Math. Sci. Mathematics, vol. 39, №.1, 2019, pp. 151-161.

[27]A.A. Huseynli, On the stability of basisness in $L_{p}(1<p<+\infty)$ of cosines and sines, Turkish Journ. Math., vol. 35, 2011, pp. 47-54.

[28]C. Bennett, R. Sharpley, Interpolation of Operators, Academic Press, 1988, 469 p.

[29]B.T. Bilalov, Some problems of approximation, Baku, Elm, 2016, 380 p. (in Russian)

[30] D.R. Adams. Morrey spaces, Springer, 2016.

[31] Kokilashvili V., Meskhi A., Rafeiro H., Samko S., Operators in non-standard function spaces, V.I. Springer, 2016.

[32] Kokilashvili V., Meshki A., Rafeiro H., Samko S., Operators in non-standard function spaces, V.II. Springer, 2016. 
Fatima Aghayar kizi Guliyeva graduated with honors from the Faculty of Mechanical-Mathematical of Baku State University, Azerbaijan in 2001. Since 2003, she has been working as a senior lecturer at the Azerbaijan University of Cooperation, and since 2008 - Deputy Dean of the Faculty of Marketing and Management. In 2011, she defended her thesis on the theme " Studying the completeness of eigen- and associated vectors of second-order operators" and received the title of Ph.D. in Mathematic. Since 2011 she has been the director of the Baku College of Business and Cooperation.

She is the author of 6 books and curricula. More than 30 scientific papers have been published in national and foreign journals.

In 2016, the International Association of Scientists, Lecturers and Professionals - The Russian Academy of Natural History awarded her with the Order of "LABORE ET SCENTIA - work and knowledge.

\section{Creative Commons Attribution License 4.0 (Attribution 4.0 International, CC BY 4.0)}

This article is published under the terms of the Creative Commons Attribution License 4.0

https://creativecommons.org/licenses/by/4.0/deed.en_US 